\title{
A Conceptual Framework of the Cooperative Analyses in Computer-Aided Engineering
}

\author{
Min-Hwan Ok ${ }^{1}$ and Tae-Soo Kwon ${ }^{2}$ \\ ${ }^{1}$ Korea Railroad Research Institute, Korea, mhok@krri.re.kr \\ ${ }^{2}$ Korea Railroad Research Institute, Korea, tskwon@krri.re.kr
}

\begin{abstract}
The role of this framework of cooperative analyses is to build a foundation for higher quality analysis in large work of CAE. The distributed vaults are constituted in peer to peer manner. A large scale analysis can be conducted in higher quality by cooperative analyses with the dividing and unifying process.
\end{abstract}

Keywords: computer-aided engineering, cooperative analysis, distributed vaults

\section{Introduction}

Computer-Aided Engineering, CAE, is nowadays a common method for analysis of some phenomena in many engineering area. Some of the CAE advantages are the cost-effectiveness, the time-efficiency, and the capability-efficacy of the worker or the working team. The capability efficacy, in this paper, is similar to the productivity of a worker or a team but dissimilar in focus on the quality than the quantity of the work produced. However this capability is intrinsically dependent on the ability of the worker or the working team, and the efficacious field or the specialty is differ between the workers or the working team.

For large work of CAE, it is desirable to divide the object into partitions and they are distributed to the workers along the person's efficacious field and then collected and unified into the entire object. In this paper we call these distributed analysis tasks cooperative analyses between the dividing and the unifying of the object. Among the partitions divided, some may have deep relevancy among them thus should be analyzed exchanging results. There have been a number of works related to cooperation including computer-aided acquisition and life-cycle support(CALS), product data management(PDM), knowledge management or etc., but none of them was suitable to this specific cooperation of analysis tasks.

Most of developments on CAE have been focused on one specific field, such as dynamic analysis, structural analysis, or fatigue analysis for example. Their interests are to pursue higher accuracy in simulations approaching to measured

Please use the following format when citing this chapter: 
values in the experimentation. However, in real world a machine is a composite of many fields from various branches. For instance, a railway system is a synthetic one of multi-body vehicle from mechanical engineering, railroad construction from civil engineering, electric power supply from electrical engineering and the other adjunct fields. In railway system, several parts have interactions between each other, for example, the wheel and the rail contacts each other and they have influences in their analyses conducted. In this work the objective of the analyses is conducted in size to be processed separately, so different from multiphysics or multidisciplinary system design optimization. After processed separately, the influencing values are exchanged and the analyses are conducted again in their sizes until some criterion is satisfied.

The role of this framework of cooperative analyses is to build a foundation for higher quality analysis in large work of CAE, in which the specialists or experts in their efficacious fields are participating. The process and system schematics are described in the next sections.

\section{Distributed Vaults of the Analysis Files}

The partitions in the cooperative analyses are distributed to, and collected from each specialist or expert. One central vault could be adequate however, for quick access of large files frequently, it is better to use local storage subsystems of experts or specialists who may be geographically dispersed. Distributed PDM contains replicated/fragmented metadata and user data since it has vaults inherent from the concept of one central vault as classified in [1].

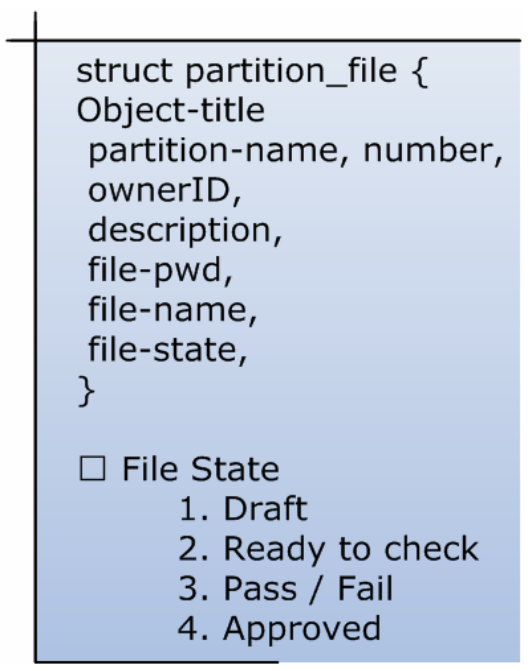

Figure 1 File organization and states. 
The file of the distributed partition is most accessed by the worker dealing with the partition. A local storage contains all the versions of the worker or the working group data, as one is shown in Figure 1, in the framework proposed in this paper.

The distributed vaults are constituted in peer to peer manner. There is no replication and thus no actions for file state change such as check in/out or release/obsolete, required by one central vault. The owner only is able to create, modify and remove the file and it is stored in the person's local storage only. A remote worker may access a cached file of the original in the person's local storage, but the cached file does not remain after the person's logout.

This distributed vaults is dissimilar from the other distributed vaults evolved from one central vault. The vaults also have one service point in which metadata is located but there is no particular server for vaulting. The original files locate only in the owner's local storage. The one service point does not have any stored file but exits for the view of conceptually one vault, which is congregated local storages in reality. The reason behind this composition of file locations is human concerns. The specialists or experts don't agree on the exposure of their knowhow by files of versions in several steps. Participating workers could endeavor to conduct more-skilled analysis concealing their know-how. In the guarantee of concealing person's know-how, the person might play an innovative role.

The cooperative analyses proceed by changing the states of files associated with partitions. After the completion of an analysis for a partition, the files appear to other remote workers indicating Ready to check. When analysis files of a relevant partition appears with Ready to check, the cooperating workers conduct larger analyses combined with files of relevant partitions and make the report of each result. The result is messaged including Pass or Fail to the owner. If the larger analysis produces the result does not satisfy a prescribed criterion, the result is messaged with Fail and additional memo describing the reason.

Only if all the results of relevant partitions are messaged with Pass, the files state is changed into Pass. Only if every file state of the relevant partitions is changed into Pass, then those files are all marked Approved.

\section{Dividing the Object into Partitions and Unifying from the Partitions}

Basically cooperative analyses proceed with the unanimous passes in peer-to-peer manner. However dividing the object, the entire work, and unifying into the entire work are processed in top-down and bottom-up manner, respectively. The dividing and unifying process is shown in Figure 2. A few partitions could be overlapped on one functional block according to the specialty of interest. Some functional blocks become partitions to be structural analysis, and some other functional blocks become partitions to be fatigue analysis. In the cooperative analysis, analyses on those partitions are conducted concurrently. Partitions the influencing values need be exchanged should Pass analyses again with the exchanged values. 
When structural analyses on every partition Pass, all the partitions are unified into one total view, the object. When every partition Passed the fatigue analysis, those are unified into another total view. With the two total views overlapped, partitions of some functional blocks may need another round of analyses due to their interactions by related features. This cross-field analysis is conducted by related teams under the co-operation of the two team leaders and the head analyst. By modified criteria after the cross-field analysis, further analyses could be necessitated in each team. In the case the object is a car, for example, an axle shaft can be a functional block. The partitions of structural analysis and the partitions of fatigue analysis may overlap on the wheel block. In Figure 3, the partition S2 and the partition F3 may overlap on some functional block, for example.

The continuation modifying the criterion involves non-deterministic rounds of analyses. This continuation is analyses in progress toward higher accuracy in simulations approaching to measured values in the experimentation. In this continuation the team leader can be replaced with a team worker, with respect to the specialty of interest. The new team leader takes over only the authority to access the metadata, and it is another reason why the files are locally stored.

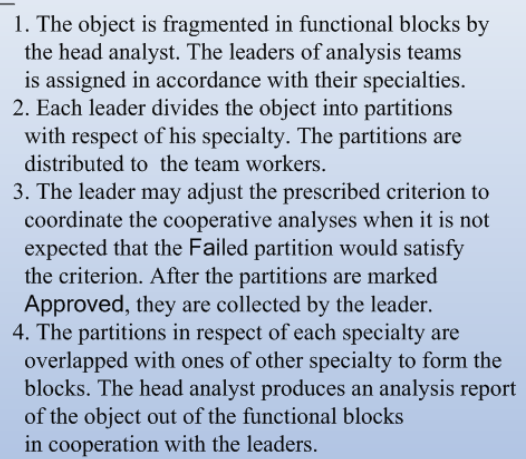

Figure 2 The dividing and unifying process.

\section{Cooperative Analyses}

The analysis results of the Approved partitions are collected and unified into the object. As each partition has partial information of the object, parametric conditions are properly prescribed. This is the prescribed criterion and settled while dividing the object by the leader and workers. The source data for analysis, such as CAD files, could be delivered via the database like described in [2]. The input data for analysis is created from the source data. It may be stored in the 
database but produced output data may not necessarily be stored in the database in this framework.

Since the prescribed criterion may not be properly settled in the first time, dividing, cooperative analyses and unifying could be repeated several times.

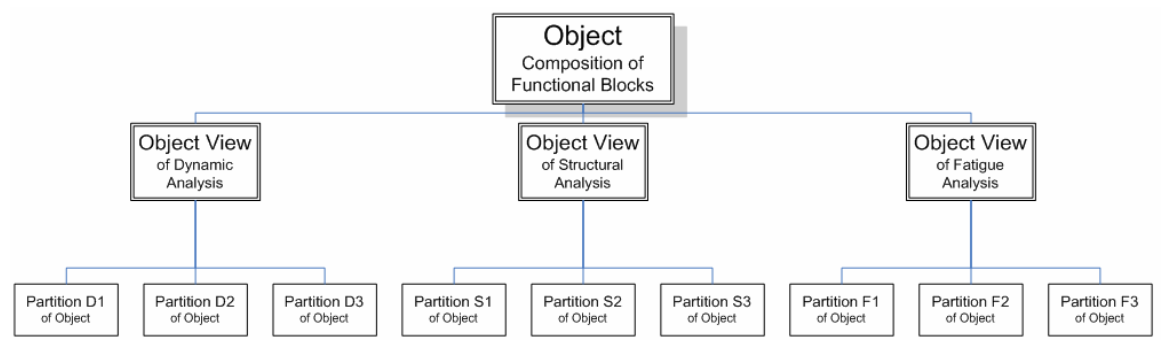

Figure 3 Dividing the object into partitions by multiple views of respective analyses.

Many of metadata structures should be required to maintain the output files, and input files as shown in Figure 1, of each partition. However those are not defined in this paper as they are application-specific. A subset of sample user interfaces is displayed in Figure 4 and 5, which are of the database for source data distribution for the analysis of a partition. The input data is created and stored at the worker's local storage.

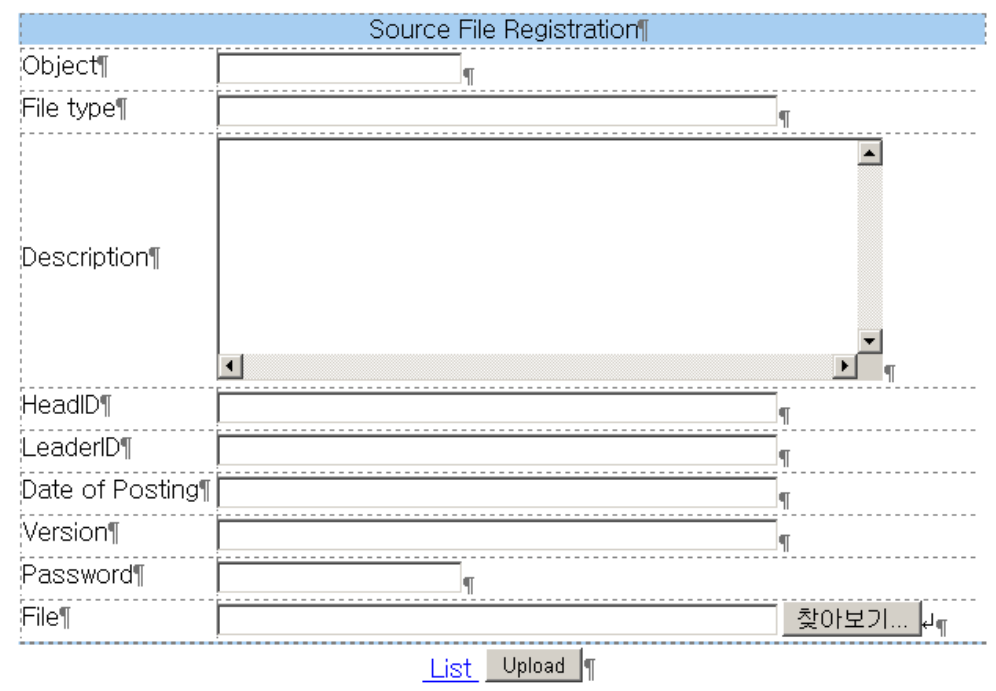

Figure 4 Source data registration for distribution. 


\section{Summary}

A peer-to-peer mannered data exchange and the process are proposed for cooperative analyses. The peer-to-peer manner eliminates tedious file check in/out and the file state field replaces the release/obsolete markings. A large scale analysis can be conducted in higher quality by cooperative analyses with the dividing and unifying process. Although this framework presents a foundation of cooperative analyses, the target of the framework is knowledge management such like [3]. However it should be avoided a complex usage from complicated process in the system. The data exchange method would evolve, in the peer-to-peer manner, for the knowledge management.

By the cooperative analyses the development of a product, with parts in higher quality, is prospective. When the knowledge management is realized in evolution of this framework, the users could derive a new technology, which is innovative, not only a better product from the knowledge management system. In this work we presented a way to collaborate on CAE with teams. In this collaborated CAE we are seeking for a practical method to derive a system for Virtual Engineering. The knowledge management would be a part of the system and the processes are refined and synthesized to be generalized ones against each sort of the analysis. While building and upgrading the cooperative analysis system, the system would develop into one for Virtual Engineering resolving the issues arising from the collaborated CAE.

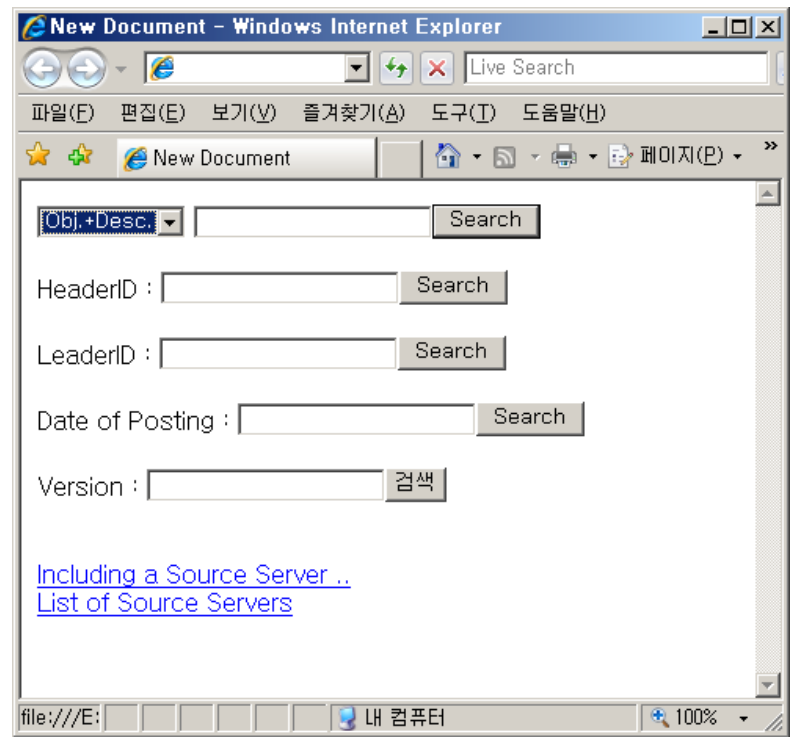

Figure 5 Search of the source data from a Web DB. 


\section{References}

1. Abramovic M., Gerhard D.: A Flexible Web-Based PDM Approach to Support Virtual Engineering Cooperation. Proc. Hawaii Int. Conf. System Sciences, USA (2000).

2. Kriegel H.-P., Müller A., Pötke M., Seidl T.: DIVE: Database Integration for Virtual Engineering. Demo. Proc. Int. Conf. Data Eng., pp. 15-16, Germany (2001).

3. Yoo S.B., Kim Y.H.: Web-based knowledge management for sharing product data in virtual enterprises. Int. J. Production Economics, Elsevier Science, vol. 75, pp. 173-183 (2002). 